A. B. Kharazishvili, Institute of Applied Mathematics, University of Tbilisi, University str. 2, 380043 Tbilisi 43, Georgia.

\title{
ON THE STEINHAUS PROPERTY FOR INVARIANT MEASURES
}

\begin{abstract}
We consider some examples of invariant measures, defined on the real line, for which an analogue of the classical Steinhaus property does not hold.
\end{abstract}

Let $\mathbb{R}$ be the real line and let $l$ be the standard Lebesgue measure on $\mathbb{R}$. It is well known that for every $l$-measurable subset $X$ of $\mathbb{R}$ the equality

$$
\lim _{h \rightarrow 0} l((X+h) \cap X)=l(X)
$$

holds. (An analogous fact is also true for a Haar measure $\mu$ defined on an arbitrary locally compact topological group $G$.) From this fact it follows immediately that if $l(X)>0$, then the difference set

$$
X-X=\{x-y: x \in X, y \in X\}
$$

contains a neighborhood of the point 0 . (See, e.g., [1, Chapter 4], or [2, p. 198].) This property of an $l$-measurable set $X$ with a strictly positive measure was first observed by Steinhaus and sometimes is called the Steinhaus property of $X$. The following result is an immediate consequence of the Steinhaus property. Let $\{X, Y\}$ be a partition of the real line into two Lebesgue measurable sets. Then at least one of the corresponding difference sets

$$
X-X, \quad Y-Y
$$

contains a neighbourhood of the point 0 and, therefore, has a nonempty interior.

Key Words: Steinhaus property, invariant measure, invariant extension of the Lebesgue measure, density point, Hamel basis

Mathematical Reviews subject classification: Primary: 28A05, 28D05

Received by the editors April 7, 1995 
On the other hand, it can be proved (although, with the aid of the Axiom of Choice) that there exists a partition $\{A, B\}$ of the real line such that the difference sets $A-A$ and $B-B$ have empty interiors. For example, such a partition $\{A, B\}$ of $\mathbb{R}$ is constructed in [3]. Note also that an analogous partition $\{A, B\}$ of $\mathbb{R}$ is considered in detail in [4]; moreover, in [4] the sets $A$ and $B$ are Bernstein subsets of $\mathbb{R}$.

Since the Steinhaus property concerns measurable sets (in the sense of Lebesgue), it is reasonable to formulate the following question. Can the sets $A$ and $B$ of a partition $\{A, B\}$ be measurable with respect to some nontrivial $\sigma$-finite invariant measures defined on the real line? In this paper we shall show that the answer is positive. In particular, we shall establish that the sets $A$ and $B$ can be measurable with respect to a certain invariant extension of Lebesgue measure $l$ (cf. [5] and [6]).

First of all we shall give a simple example of a partition $\{A, B\}$ of $\mathbb{R}$ having the property that $\operatorname{int}(A-A)=\emptyset$ and $\operatorname{int}(B-B)=\emptyset$.

Example. Let us consider the real line $\mathbb{R}$ as a vector space $E$ over the field $\mathbb{Q}$ of all rational numbers. Take the one-element subset $\{1\}$ of the space $E$ and extend this subset to a Hamel basis $H$ of $E$. Denote by $\Gamma$ the vector subspace of the space $E$ generated by the set $H \backslash\{1\}$. In fact, $\Gamma$ is a vector hyperplane in $E$. Obviously, we can represent $E=\mathbb{R}$ as the direct sum of $\mathbb{Q}$ and $\Gamma$, i.e. we have $E=\mathbb{Q}+\Gamma, \quad \mathbb{Q} \cap \Gamma=\{0\}$. Furthermore, it is clear that every rational number $q$ can be uniquely represented in the form

$$
q=n(q)+t(q)
$$

where $n(q)$ is an integer and $0 \leq t(q)<1$. Put

$$
\begin{aligned}
& Q_{1}=\text { the set of all } q \text { for which } n(q) \text { is an odd number; } \\
& Q_{2}=\text { the set of all } q \text { for which } n(q) \text { is a even number. }
\end{aligned}
$$

Evidently, we get a partition $\left\{Q_{1}, Q_{2}\right\}$ of the set $\mathbb{Q}$. Define

$$
A=Q_{1}+\Gamma, \quad B=Q_{2}+\Gamma .
$$

Then $\{A, B\}$ is a partition of the real line and the difference sets $A-A$ and $B-B$ have empty interiors. Indeed, it is easy to check that

$$
\begin{gathered}
A-A=\left(Q_{1}-Q_{1}\right)+\Gamma, \\
B-B=\left(Q_{2}-Q_{2}\right)+\Gamma, \\
1 \notin Q_{1}-Q_{1}, \quad 1 \notin Q_{2}-Q_{2} .
\end{gathered}
$$


Hence, we obtain

$$
\begin{aligned}
& (A-A) \cap(1+\Gamma)=\emptyset, \\
& (B-B) \cap(1+\Gamma)=\emptyset .
\end{aligned}
$$

Taking into account the fact that $\Gamma$ is an everywhere dense subgroup of the additive group of $\mathbb{R}$, we get that $1+\Gamma$ is an everywhere dense subset of $\mathbb{R}$ and, consequently, we have

$$
\operatorname{int}(A-A)=\emptyset, \quad \operatorname{int}(B-B)=\emptyset .
$$

Moreover, we can choose a Hamel basis $H$ in such a way that the hyperplane $\Gamma$ would be a Bernstein subset of the real line $\mathbb{R}$. In this case $1+\Gamma$ is also a Bernstein subset of $\mathbb{R}$, and we see that $A, B, A-A$ and $B-B$ are Bernstein sets also.

Furthermore, it is not difficult to show that the hyperplane $\Gamma$ is always an $l$-thick subset of $\mathbb{R}$, i.e. the inner Lebesgue measure of the set $\mathbb{R} \backslash \Gamma$ is equal to zero. Let us consider the family of all distinct translates of $\Gamma$. Obviously, this family is countable. Denote it by $\left\{\Gamma_{n}: n \in \omega\right\}$. It is clear that $\Gamma_{n} \cap \Gamma_{m}=\emptyset$ if $n \neq m$. Take the class $S$ of all subsets $Z$ of $\mathbb{R}$ which can be represented in the form

$$
Z=\cup\left\{\Gamma_{n} \cap X_{n}: n \in \omega\right\},
$$

where $\left\{X_{n}: n \in \omega\right\}$ is a countable family of $l$-measurable sets in $\mathbb{R}$. It is easy to check that $S$ is a $\sigma$-algebra of sets and, moreover, $S$ is invariant under the group of all translations of $\mathbb{R}$. Now, for each $Z \in S$, let us put

$$
\nu(Z)=l\left(X_{0}\right)+l\left(X_{1}\right)+\ldots+l\left(X_{n}\right)+\ldots .
$$

Since all the sets $\Gamma_{n}(n \in \omega)$ are $l$-thick and pairwise disjoint, the functional $\nu$ is well-defined on the $\sigma$-algebra $S$. Also it is not difficult to check that the following relations are fulfilled.

1) $\nu$ is a non-atomic $\sigma$-finite measure on $\mathbb{R}$.

2) $\nu$ is invariant under the group of all translations of $\mathbb{R}$ (moreover, $\nu$ is invariant under the group of all isometric transformations of $\mathbb{R}$ ).

3) $\operatorname{dom}(l) \subset \operatorname{dom}(\nu)$.

4) $\Gamma \in \operatorname{dom}(\nu), \quad A \in \operatorname{dom}(\nu), \quad B \in \operatorname{dom}(\nu)$.

5) $\nu(A)=\nu(B)=+\infty$. 
Thus, we see that for two $\nu$-measurable sets $A$ and $B$ the Steinhaus property does not hold, while $\{A, B\}$ is a partition of the real line.

We remark also that the $\nu$-measurable set $\Gamma$ is a Vitali subset of the real line, because for each $h \in \mathbb{R}$ we have $\operatorname{card}((\mathbb{Q}+h) \cap \Gamma)=1$. This unusual property of the measure $\nu$ was mentioned in [5]. Of course, such a measure $\nu$ cannot be an invariant extension of Lebesgue measure $l$, since a certain Vitali set belongs to the domain of $\nu$. Note that if $X$ is an arbitrary Lebesgue measurable subset of the real line satisfying the inequality $l(X)>0$, then $\nu(X)=+\infty$. From this fact it also follows that the measure $\nu$ cannot extend the Lebesgue measure $l$.

Now let us consider an arbitrary measure $\mu$ on $\mathbb{R}$ extending $l$ and invariant under the group of all translations of $\mathbb{R}$. Take any partition $\{A, B\}$ of $\mathbb{R}$ such that $A \in \operatorname{dom}(\mu)$. (Hence, $B \in \operatorname{dom}(\mu)$ also.) The following question arises in a natural way. Is it true that at least one of the difference sets $A-A$ and $B-B$ has a nonempty interior? We shall show below that the answer to this question is negative. But first we consider a situation where we can answer the posed question in the affirmative.

It is easy to see that, for Lebesgue measure $l$, the Steinhaus property may be obtained as a direct consequence of the classical Lebesgue theorem on density points of $l$-measurable sets. More generally, we have the following

Proposition 1 Let $\mu$ be a measure on $\mathbb{R}$ extending $l$ and invariant under the group of all translations of $\mathbb{R}$. Let $\{A, B\}$ be a partition of $\mathbb{R}$ consisting of two $\mu-$ measurable sets. Suppose also that there exists a segment $I \subset \mathbb{R}$ such that $\mu(I \cap A) \neq l(I) / 2$. Then at least one of the difference sets $A-A$ and $B-B$ contains a neighborhood of the point 0 . (Hence, at least one of these difference sets has a nonempty interior.)

Proof. Obviously, we can write $l(I)=\mu(I)=\mu(I \cap A)+\mu(I \cap B)$ and, consequently, $\mu(I \cap A)>l(I) / 2$ or $\mu(I \cap B)>l(I) / 2$. Without loss of generality we may assume that $\mu(I \cap A)>l(I) / 2$. Let us show that in this case the difference set $A-A$ contains a neighbourhood of the point 0 . Indeed, suppose that $A-A$ does not contain an open interval with the center 0 . Then there exists a sequence $\left\{h_{n}: n \in \omega\right\}$ of elements of $\mathbb{R}$ satisfying the relations

$$
\begin{aligned}
\lim _{n \rightarrow \infty} h_{n} & =0, \\
\left(h_{n}+A\right) \cap A & =\emptyset \quad(n \in \omega) .
\end{aligned}
$$

It is clear that, for some $\epsilon>0$, we have $\mu(I \cap A)>l(I) / 2+\epsilon$. Let $n$ be a natural 
number such that $\mu\left(I \cup\left(h_{n}+I\right)\right)<l(I)+\epsilon$. Then we get the inequalities

$$
\begin{aligned}
l(I)+\epsilon & >\mu\left(I \cup\left(h_{n}+I\right)\right) \\
& \geq \mu\left((I \cap A) \cup\left(\left(h_{n}+I\right) \cap\left(h_{n}+A\right)\right)\right) \\
& =2 \mu(I \cap A)>l(I)+2 \epsilon,
\end{aligned}
$$

which give us a contradiction.

Proposition 1 shows that if we want to construct a partition $\{A, B\}$ of $\mathbb{R}$ consisting of $\mu$-measurable sets such that $\operatorname{int}(A-A)=\operatorname{int}(B-B)=\emptyset$, then we necessarily must have $\mu(I \cap A)=\mu(I \cap B)=l(I) / 2$, for every segment $I$ on the real line $\mathbb{R}$. From the last equalities it immediately follows also that

$$
\mu(X \cap A)=\mu(X \cap B)=l(X) / 2,
$$

for every $l$-measurable subset $X$ of $\mathbb{R}$ (note that several examples of invariant extensions of $l$ satisfying the last relation are investigated in [6, p. 117]).

Now we shall construct a measure $\mu$ and a partition $\{A, B\}$ with the properties mentioned above.

Let $\mathbf{T}$ denote the one-dimensional torus, i.e. put

$$
\mathbf{T}=\left\{(x, y) \in \mathbb{R}^{2}: x^{2}+y^{2}=1\right\} .
$$

Obviously, $\mathbf{T}$ is a commutative divisible compact topological group with respect to the standard structures on $\mathbf{T}$. It is also clear that we may consider $\mathbf{T}$ as a closed interval $[0,2 \pi]$ in which the end-points 0 and $2 \pi$ are identified. Equip $\mathbf{T}$ with a probability Lebesgue measure $\lambda$ invariant under the group of all rotations of $\mathbf{T}$. Now we apply the method similar to the method of a well known paper [7], where a nonseparable invariant extension of Lebesgue measure is constructed. Let $\left\{h_{n}: n \in \omega\right\}$ be an arbitrary countable, everywhere dense subset of $\mathbb{R}$ linearly independent over the field $\mathbb{Q}$ of all rational numbers. Using the method of transfinite recursion we can define a homomorphism $f$ from the abstract group $\mathbb{R}$ into the abstract group $\mathbf{T}$ such that

1) the graph of $f$, i.e. the set $\{(x, f(x)): x \in \mathbb{R}\}$, is an $(l \times \lambda)$-thick subset of the product space $\mathbb{R} \times \mathbf{T}$. (In other words, the inner $(l \times \lambda)$-measure of the complement of this graph is equal to zero.);

2) $f\left(h_{n}\right)=\pi$ for each $n \in \omega$.

The construction of the homomorphism $f$ with properties 1) and 2) is standard and does not present any difficulties. In fact, we use here a general theorem of the theory of commutative groups which states that any partial homomorphism from a commutative group $G_{1}$ into a divisible commutative group $G_{2}$ can be extended to a homomorphism from $G_{1}$ into $G_{2}{ }^{1}$. This theo-

\footnotetext{
${ }^{1}$ (see, e.g., A. G. Kurosh, The Theory of Groups, "Nauka", Moscow, 1967, p. 551 - 552)
} 
rem has many useful corollaries; for example,

a) any commutative group can be embedded in a divisible commutative group;

b) any infinite commutative group admits a nontrivial topologization;

c) any commutative group is algebraically isomorphic with an everywhere dense subgroup of a commutative compact topological group.

Now, starting with the homomorphism $f$ mentioned above, we can define the required invariant extension $\mu$ of the measure $l$. Namely, for each $(l \times \lambda)-$ measurable set $Z$, let us put

$$
Z^{*}=\{x \in \mathbb{R}:(x, f(x)) \in Z\} .
$$

Furthermore, put

$$
\begin{gathered}
S=\left\{Z^{*}: Z \in \operatorname{dom}(l \times \lambda)\right\}, \\
\mu\left(Z^{*}\right)=(l \times \lambda)(Z) \quad\left(Z^{*} \in S\right) .
\end{gathered}
$$

It is not difficult to check that $S$ is a $\sigma$-algebra of subsets of $\mathbb{R}$ and the functional $\mu$ is well-defined on $S$. It can be also checked that $\mu$ is a measure on this $\sigma$-algebra. Moreover, $\mu$ extends $l$ and is invariant under the group of all isometric transformations of $\mathbb{R}$. As remarked in [8], the Steinhaus property does not hold for such a measure $\mu$. More exactly, let us put

$$
\begin{gathered}
A=\{x \in \mathbb{R}:(x, f(x)) \in \mathbb{R} \times[0, \pi)\}, \\
B=\{x \in \mathbb{R}:(x, f(x)) \in \mathbb{R} \times[\pi, 2 \pi)\} .
\end{gathered}
$$

From the definition of the sets $A$ and $B$ it immediately follows that

(1) $\{A, B\}$ is a partition of the real line $\mathbb{R}$;

(2) $A$ and $B$ are $\mu$-measurable subsets of $\mathbb{R}$;

(3) $\mu(A)=\mu(B)=+\infty$;

(4) $h_{n}+A=B$ and $h_{n}+B=A$ for each $n \in \omega$.

In particular, we have

$$
\left(h_{n}+A\right) \cap A=\emptyset, \quad\left(h_{n}+B\right) \cap B=\emptyset
$$

for all $n \in \omega$. Hence, taking into account the fact that $\left\{h_{n}: n \in \omega\right\}$ is an everywhere dense subset of $\mathbb{R}$, we obtain that the difference sets $A-A$ and $B-B$ have empty interiors.

Slightly changing the above argument we can get a more general result. Namely, we have the following 
Proposition 2 There exists a measure $\mu$ on the real line $\mathbb{R}$, extending $l$ and invariant under the group of all isometric transformations of $\mathbb{R}$, and a partition $\{A, B\}$ of $\mathbb{R}$ consisting of two $\mu$-measurable sets such that all the sets $A, B, A-$ $A$ and $B-B$ are totally imperfect subsets of $\mathbb{R}$, i.e. they are Bernstein subsets of $\mathbb{R}$.

Finally, let us recall that the partition $\{A, B\}$ has also the following property. For every segment $I$ on the real line $\mathbb{R}$, the equalities

$$
\mu(I \cap A)=\mu(I \cap B)=l(I) / 2
$$

are fulfilled. Note in connection with this fact that a much stronger property of some subsets of the real line is discussed in detail in the paper [9]. For other properties of the measure $\mu$ constructed above, see [8].

\section{References}

[1] J. C. Oxtoby, Measure and Category, Springer Verlag, Berlin, 1971.

[2] J. C. Morgan II, Point Set Theory, Marcel Dekker, Inc., New York and Basel, 1990.

[3] Harry I. Miller, Some decomposition theorems for the real line, Radovi Matematicki, 1 (1985), 31-37.

[4] B. King, Some remarks on difference sets of Bernstein sets, Real Analysis Exchange, 19 no. 2 (1993-1994), 478-490.

[5] A. B. Kharazishvili, Some applications of Hamel bases, Bull. Acad. Sci. Georgian SSR, 85 no. 1 (1977), 17-20, (in Russian).

[6] A. B. Kharazishvili, Invariant Extensions of the Lebesgue Measure, Izd. Tbil. Gos. Univ., Tbilisi, 1983, (in Russian).

[7] K. Kodaira, S. Kakutani, A nonseparable translation invariant extension of the Lebesgue measure space, Ann. Math., 52 (1950), 574-579.

[8] A. B. Kharazishvili, Some remarks on density points and the uniqueness property for invariant extensions of the Lebesgue measure, Acta Universitatis Carolinae - Mathematica et Physica, 35 no. 2 (1994), 33-39.

[9] R. D. Mabry, Sets which are well-distributed and invariant relative to all isometry invariant total extensions of Lebesgue measure, Real Analysis Exchange, 16 (1990-1991), 425-459. 